Pacific

Journal of

Mathematics

HAMILTONIAN-MINIMAL LAGRANGIAN SUBMANIFOLDS IN COMPLEX SPACE FORMS

ILDEFONSO CASTRO, HAIZHONG LI AND FRANCISCO URBANO 


\title{
HAMILTONIAN-MINIMAL LAGRANGIAN SUBMANIFOLDS IN COMPLEX SPACE FORMS
}

\author{
Ildefonso CASTRO, HAIZHONG Li AND FRANCISCO URBANO
}

\begin{abstract}
Using Legendrian immersions and, in particular, Legendre curves in odddimensional spheres and anti-de Sitter spaces, we construct new examples of Hamiltonian-minimal Lagrangian submanifolds in complex projective and hyperbolic spaces, including explicit one-parameter families of embeddings of quotients of certain product manifolds. We also give new examples of minimal Lagrangian submanifolds in complex projective and hyperbolic spaces. Making use of all these constructions, we get Hamiltonian-minimal and special Lagrangian cones in complex Euclidean space as well.
\end{abstract}

\section{Introduction}

Let $\left(\tilde{M}^{n}, J,\langle\rangle,\right)$ be a Kähler manifold of complex dimension $n$, where $J$ is the complex structure and $\langle$,$\rangle the Kähler metric. The Kähler 2-form is defined by$ $\omega(\cdot, \cdot)=\langle J \cdot, \cdot\rangle$. An immersion $\psi: M^{n} \rightarrow \tilde{M}^{n}$ of an $n$-dimensional manifold $M$ is called Lagrangian if $\psi^{*} \omega \equiv 0$. For this type of immersions, $J$ defines a bundle isomorphism between the tangent bundle $T M$ and the normal bundle $T^{\perp} M$.

A vector field $X$ on $\widetilde{M}$ is a Hamiltonian vector field if there exists a smooth function $F: \widetilde{M} \rightarrow \mathbb{R}$ such that $X=J \widetilde{\nabla} F$, where $\widetilde{\nabla}$ is the gradient in $\widetilde{M}$. The diffeomorphisms of the flux of a Hamiltonian vector field transform Lagrangian submanifolds into Lagrangian ones.

In this setting, Oh [1990] studied the following natural variational problem. A normal vector field $\xi$ to a Lagrangian immersion $\psi: M^{n} \rightarrow \widetilde{M}^{n}$ is called Hamiltonian if $\xi=J \nabla f$, where $f \in C^{\infty}(M)$ and $\nabla f$ is the gradient of $f$ with respect to the induced metric. Take $f \in C_{0}^{\infty}(M)$ and let $\left\{\psi_{t}: M \rightarrow \tilde{M}\right\}$ be a variation of $\psi$, with $\psi_{0}=\psi$ and $\left.\frac{d}{d t}\right|_{t=0} \psi_{t}=\xi$. The first variation of the volume functional is given by

$$
\left.\frac{d}{d t}\right|_{t=0} \operatorname{vol}\left(M, \psi_{t}^{*}\langle,\rangle\right)=-\int_{M} f \operatorname{div} J H d M
$$

MSC2000: primary 53C42, 53B25; secondary 53A05, 53D12.

Keywords: Hamiltonian-minimal, Lagrangian submanifolds, Legendrian immersions.

Castro's and Urbano's research is partially supported by a MEC-Feder grant MTM2004-00109.

Li is partially supported by Zhongdian grant No. 10531090 of NSFC.. 
(see [Oh 1990]), where $H$ is the mean curvature vector of the immersion $\psi$ and div denotes the divergence operator on $M$. Oh called the critical points of this variational problem Hamiltonian minimal (or H-minimal) Lagrangian submanifolds; they are characterized by the third-order differential equation $\operatorname{div} J H=0$. In particular, minimal Lagrangian submanifolds (where "minimal" means that the mean curvature vector vanishes) are trivially $\mathrm{H}$-minimal; so is, more generally, any Lagrangian submanifold with parallel mean curvature vector.

Even when $\widetilde{M}$ is a simply connected complex space form, only few examples of H-minimal Lagrangian submanifolds are known outside the class of Lagrangian submanifolds with parallel mean curvature vector.

This can be a brief history of them: $\mathbb{S}^{1}$-invariant H-minimal Lagrangian tori in the complex Euclidean plane $\mathbb{C}^{2}$ were classified in [Castro and Urbano 1998]. H-minimal Lagrangian cones in $\mathbb{C}^{2}$ were studied in [Schoen and Wolfson 1999]. Hélein and Romon [2000; 2002a] derived a Weierstrass-type representation formula to describe all H-minimal Lagrangian tori and Klein bottles in $\mathbb{C}^{2}$. When the ambient space is the complex projective plane $\mathbb{C P}^{2}$ or the complex hyperbolic plane $\mathbb{C} \mathbb{H}^{2}$, conformal parametrizations of H-minimal Lagrangian surfaces using holomorphic data were obtained in [Hélein and Romon 2002b; 2003]. Making use of this technique, Anciaux [2003] constructed H-minimal Lagrangian singly periodic cylinders and $\mathrm{H}$-minimal Lagrangian surfaces with a nonconical singularity in $\mathbb{C}^{2}$. Only recently have examples of $\mathrm{H}$-minimal Lagrangian submanifolds of arbitrary dimension in $\mathbb{C}^{n}$ and $\mathbb{C} \mathbb{P}^{n}$ been found, in [Mironov 2004]. A classification of H-minimal Lagrangian submanifolds foliated by $(n-1)$-spheres in $\mathbb{C}^{n}$ is given in [Anciaux et al. 2006].

Our aim in this paper is the construction of $\mathrm{H}$-minimal Lagrangian submanifolds in complex Euclidean space $\mathbb{C}^{n}$, complex projective space $\mathbb{C P}^{n}$ and complex hyperbolic space $\mathbb{C} \mathbb{H}^{n}$, for arbitrary $n \geq 2$. The examples in $\mathbb{C} \mathbb{P}^{n}$ are constructed by projection, via the Hopf fibration $\Pi: \mathbb{S}^{2 n+1} \rightarrow \mathbb{C P}^{n}$, of certain family of Legendrian submanifolds of the sphere $\mathbb{S}^{2 n+1}$ (Corollary 3.2). The cones with links in this family of Legendrian submanifolds provide new examples of H-minimal Lagrangian submanifolds in $\mathbb{C}^{n+1}$ (Section 5). Using the Hopf fibration $\Pi: \mathbb{H}_{1}^{2 n+1} \rightarrow \mathbb{C} \mathbb{H}^{n}$ and a similar family of Legendrian submanifolds of the anti-de Sitter space $\mathbb{U}_{1}^{2 n+1}$ (Corollary 6.5), we also find examples of H-minimal Lagrangian submanifolds in $\mathbb{C} \mathbb{H}^{n}$. In a certain sense, our construction is reminiscent of the Smith join method (see [Eells and Ratto 1993]) for constructing harmonic maps between spheres.

In $\mathbb{C P}^{n}$, we emphasize two different one-parameter families of $\mathrm{H}$-minimal Lagrangian immersions described in Corollaries 4.1 and 4.4; as a particular case, in Corollary 4.2 we provide explicit Lagrangian H-minimal embeddings of certain quotients of $\mathbb{S}^{1} \times \mathbb{S}^{n_{1}} \times \mathbb{S}^{n_{2}}$, where $n_{1}+n_{2}+1=n$. 
In $\mathbb{C} \mathbb{G}^{n}$, we also point out in Corollary 6.6 a one-parameter family of $\mathrm{H}$-minimal Lagrangian immersions, which (in the easiest cases) induce explicit Lagrangian $\mathrm{H}$ minimal embeddings of certain quotients of $\mathbb{S}^{1} \times \mathbb{S}^{n_{1}} \times \mathbb{R} \mathbb{R}^{n_{2}}$, for $n_{1}+n_{2}+1=n$ (see Corollary 6.7). Here $\mathbb{R} \mathbb{H}^{n_{2}}$ denotes real hyperbolic space.

As a byproduct, using our method of construction, we also obtain new examples of minimal Lagrangian submanifolds in $\mathbb{C P}^{n}$ (Corollary 4.1, Remark 4.3 and Corollary 4.4) and $\mathbb{C} \mathbb{E}^{n}$ (Corollaries 6.5 and 6.9), as well as special Lagrangian cones in $\mathbb{C}^{n+1}$ (see Section 5).

\section{Lagrangian submanifolds versus Legendrian submanifolds}

Let $\mathbb{C}^{n+1}$ be complex Euclidean space endowed with the Euclidean metric $\langle$,$\rangle and$ standard complex structure $J$. The Liouville 1-form is given by $\Lambda_{z}(v)=\langle v, J z\rangle$ for all $z \in \mathbb{C}^{n+1}$ and all $v \in T_{z} \mathbb{C}^{n+1}$, and the Kähler 2-form is $\omega=d \Lambda / 2$. We denote the $(2 n+1)$-dimensional unit sphere in $\mathbb{C}^{n+1}$ by $\mathbb{S}^{2 n+1}$ and by $\Pi: \mathbb{S}^{2 n+1} \rightarrow \mathbb{C} \mathbb{P}^{n}$, $\Pi(z)=[z]$, the Hopf fibration of $\mathbb{S}^{2 n+1}$ on the complex projective space $\mathbb{C} \mathbb{P}^{n}$. We denote the Fubini-Study metric, the complex structure and the Kähler two-form in $\mathbb{C P}^{n}$ by $\langle\rangle,$,$J and \omega$. This metric has constant holomorphic sectional curvature 4 .

We will also denote by $\Lambda$ the restriction to $\mathbb{S}^{2 n+1}$ of the Liouville 1 -form of $\mathbb{C}^{n+1}$. So $\Lambda$ is the contact 1 -form of the canonical Sasakian structure on the sphere $\mathbb{S}^{2 n+1}$. An immersion $\phi: M^{n} \rightarrow \mathbb{S}^{2 n+1}$ of an $n$-dimensional manifold $M$ is said to be Legendrian if $\phi^{*} \Lambda \equiv 0$. In this case $\phi$ is isotropic in $\mathbb{C}^{n+1}$, that is, $\phi^{*} \omega \equiv 0$; in particular, the normal bundle $T^{\perp} M$ splits as $J(T M) \oplus \operatorname{span}\{J \phi\}$. This means that $\phi$ is horizontal with respect to the Hopf fibration $\Pi: \mathbb{S}^{2 n+1} \rightarrow \mathbb{C P}^{n}$, and hence $\Phi=\Pi \circ \phi: M^{n} \rightarrow \mathbb{C P}^{n}$ is a Lagrangian immersion and the metrics induced on $M^{n}$ by $\phi$ and $\Phi$ are the same. It is easy to check that $J \phi$ is a totally geodesic normal vector field, so the second fundamental forms of $\phi$ and $\Phi$ are related by

$$
\Pi_{*}\left(\sigma_{\phi}(v, w)\right)=\sigma_{\Phi}\left(\Pi_{*} v, \Pi_{*} w\right) \quad \text { for all } v, w \in T M
$$

Thus the mean curvature vector $H$ of $\phi$ satisfies $\langle H, J \phi\rangle=0$. In particular, $\phi$ : $M^{n} \rightarrow \mathbb{S}^{2 n+1}$ is minimal if and only if $\Phi=\Pi \circ \phi: M^{n} \rightarrow \mathbb{C} \mathbb{P}^{n}$ is minimal.

In this way, we can construct (minimal) Lagrangian submanifolds in $\mathbb{C P}^{n}$ by projecting (minimal) Legendrian manifolds in $\mathbb{S}^{2 n+1}$ via the Hopf fibration $\Pi$.

Conversely, any Lagrangian immersion $\Phi: M^{n} \rightarrow \mathbb{C P}^{n}$ has a local horizontal lift to $\mathbb{S}^{2 n+1}$ with respect to the Hopf fibration $\Pi$; this local lift is unique up to rotations. Only Lagrangian immersions in $\mathbb{C P}^{n}$ have such lifts.

In this article we construct examples of Lagrangian submanifolds of $\mathbb{C P}^{n}$ by constructing examples of Legendrian submanifolds of $\mathbb{S}^{2 n+1}$. We start with some geometric properties of Legendrian submanifolds in $\mathbb{S}^{2 n+1}$. 
Let $\Omega$ be the complex $n$-form on $\mathbb{S}^{2 n+1}$ given by

$$
\Omega_{z}\left(v_{1}, \ldots, v_{n}\right)=\operatorname{det}_{\mathbb{C}}\left\{z, v_{1}, \ldots, v_{n}\right\} .
$$

If $\phi: M^{n} \rightarrow \mathbb{S}^{2 n+1}$ is a Legendrian immersion of a manifold $M$, then $\phi^{*} \Omega$ is a complex $n$-form on $M$. In the next result we analyze this $n$-form $\phi^{*} \Omega$.

Lemma 2.1. If $\phi: M^{n} \rightarrow \mathbb{S}^{2 n+1}$ is a Legendrian immersion of a manifold $M$, then

$$
\nabla\left(\phi^{*} \Omega\right)=\alpha_{H} \otimes \phi^{*} \Omega,
$$

where $\alpha_{H}$ is the one-form on $M$ defined by $\alpha_{H}(v)=n i\langle H, J v\rangle$ and $H$ is the mean curvature vector of $\phi$. Consequently, $M$ is orientable if $\phi$ is minimal.

Proof. Let $\left\{E_{1}, \ldots, E_{n}\right\}$ be an orthonormal frame on an open subset $U \subset M$ containing $p$, such that $\nabla_{v} E_{i}=0$ for all $v \in T_{p} M$ and $i=1, \ldots, n$. We define $A: U \rightarrow U(n+1)$ by $A=\left\{\phi, \phi_{*}\left(E_{1}\right), \ldots, \phi_{*}\left(E_{n}\right)\right\}$. Then

$$
\left(\nabla_{v} \phi^{*} \Omega\right)\left(E_{1}, \ldots, E_{n}\right)=v\left(\operatorname{det}_{\mathbb{C}} A\right)=\operatorname{det}_{\mathbb{C}} A \operatorname{Trace}\left(v(A) \bar{A}^{t}\right),
$$

where $\bar{A}^{t}$ denotes the transpose conjugate matrix of $A$. We easily see that $v(A)=\left\{\phi_{*}(v), \sigma_{\phi}\left(v, E_{1}(p)\right)-\left\langle v, E_{1}(p)\right\rangle \phi, \ldots, \sigma_{\phi}\left(v, E_{n}(p)\right)-\left\langle v, E_{n}(p)\right\rangle \phi\right\}$, and so we deduce that

$$
\left(\nabla_{v} \phi^{*} \Omega\right)\left(E_{1}(p), \ldots, E_{n}(p)\right)=n i\langle H(p), J v\rangle\left(\phi^{*} \Omega\right)\left(E_{1}, \ldots, E_{n}\right)(p) .
$$

Using this in the preceding expression we get the result.

Suppose that our Legendrian submanifold $M$ is oriented. Then we can consider the well defined map $\beta: M^{n} \rightarrow \mathbb{R} / 2 \pi \mathbb{Z}$ given by

$$
e^{i \beta(p)}=\left(\phi^{*} \Omega\right)_{p}\left(e_{1}, \ldots, e_{n}\right),
$$

where $\left\{e_{1}, \ldots, e_{n}\right\}$ is an oriented orthonormal frame in $T_{p} M$. We will call $\beta$ the Legendrian angle map of $\phi$. As a consequence of (1) we obtain

$$
J \nabla \beta=n H,
$$

and so we deduce:

Proposition 2.2. A Legendrian immersion $\phi: M^{n} \rightarrow \mathbb{S}^{2 n+1}$ of an oriented manifold $M$ is minimal if and only if the Legendrian angle map $\beta$ of $\phi$ is constant.

A vector field $X$ on $\mathbb{S}^{2 n+1}$ is a contact vector field if $\mathscr{L}_{X} \Lambda=g \Lambda$, for some function $g \in C^{\infty}\left(\mathbb{S}^{2 n+1}\right)$, where $\mathscr{L}$ is the Lie derivative in $\mathbb{S}^{2 n+1}$. As shown in [McDuff and Salamon 1998], for instance, $X$ is a contact vector field if and only if there exists $F \in C^{\infty}\left(\mathbb{S}^{2 n+1}\right)$ such that

$$
X_{z}=J(\bar{\nabla} F)_{z}+2 F J z, \quad z \in \mathbb{S}^{2 n+1},
$$


where $\bar{\nabla} F$ is the gradient of $F$. The diffeomorphisms of the flux $\left\{\varphi_{t}\right\}$ of $X$ are contactomorphisms of $\mathbb{S}^{2 n+1}$, that is, $\varphi_{t}^{*} \Lambda=e^{h_{t}} \Lambda$, and so they transform Legendrian submanifolds into same. The Lie algebra of the group of contactomorphisms of $\mathbb{S}^{2 n+1}$ is the space of contact vector fields. In this setting, it is natural to study the following variational problem.

Let $\phi: M^{n} \rightarrow \mathbb{S}^{2 n+1}$ a Legendrian immersion with mean curvature vector $H$. A normal vector field $\xi_{f}$ to $\phi$ is called a contact field if

$$
\xi_{f}=J \nabla f+2 f J \phi
$$

where $f \in C^{\infty}(M)$ and $\nabla f$ is the gradient of $f$ with respect to the induced metric. If $f \in C_{0}^{\infty}(M)$ and $\left\{\phi_{t}: M \rightarrow \mathbb{S}^{2 n+1}\right\}$ is a variation of $\phi$ with $\phi_{0}=\phi$ and $\left.\frac{d}{d t}\right|_{t=0} \phi_{t}=$ $\xi_{f}$, the first variation of the volume functional is given by

$$
\left.\frac{d}{d t}\right|_{t=0} \operatorname{vol}\left(M, \phi_{t}^{*}\langle,\rangle\right)=-\int_{M}\left\langle H, \xi_{f}\right\rangle d M
$$

But using Stokes' Theorem,

$$
\begin{aligned}
\int_{M}\left\langle H, \xi_{f}\right\rangle d M & =\int_{M}\langle H, J \nabla f+2 f J \phi\rangle d M \\
& =-\int_{M}\langle J H, \nabla f\rangle d M=\int_{M} f \operatorname{div} J H d M
\end{aligned}
$$

This means that the critical points of the above variational problem are Legendrian submanifolds such that

$$
\operatorname{div} J H=0 .
$$

Definition 2.3. A Legendrian immersion $\phi: M^{n} \rightarrow \mathbb{S}^{2 n+1}$ is said to be contact minimal (or briefly $C$-minimal) if it is a critical point of the preceding variational problem, that is, if $\operatorname{div} J H=0$.

Clearly, minimal Legendrian submanifolds and Legendrian submanifolds with parallel mean curvature vector are C-minimal. As a consequence of (2) and the geometric relationship between Legendrian and Lagrangian submanifolds mentioned at the beginning of this section, we get:

Proposition 2.4. Let $\phi: M^{n} \rightarrow \mathbb{S}^{2 n+1}$ be a Legendrian immersion of a Riemannian manifold $M$.

(1) If $M$ is oriented, $\phi$ is $C$-minimal if and only if the Legendrian angle $\beta$ of $\phi$ is a harmonic map.

(2) $\phi$ is $C$-minimal if and only if $\Phi=\Pi \circ \phi: M^{n} \rightarrow \mathbb{C P}^{n}$ is H-minimal. 


\section{A new construction of C-minimal Legendrian immersions}

After Proposition 2.4, it is clear that constructing C-minimal Legendrian immersions in odd-dimensional spheres is a good way to find H-minimal Lagrangian submanifolds in $\mathbb{C} \mathbb{P}^{n}$. This is the purpose of this section.

Let $n_{1}, n_{2} \geq 0$ be integers with $n=n_{1}+n_{2}+1$. The product $\mathrm{SO}\left(n_{1}+1\right) \times$ $\mathrm{SO}\left(n_{2}+1\right)$ of special orthogonal groups acts on $\mathbb{S}^{2 n+1} \subset \mathbb{C}^{n+1}$ as a subgroup of isometries:

$$
\left(A_{1}, A_{2}\right) \in \mathrm{SO}\left(n_{1}+1\right) \times \mathrm{SO}\left(n_{2}+1\right) \mapsto\left(\begin{array}{c|c}
A_{1} & \\
\hline & A_{2}
\end{array}\right) \in \mathrm{SO}(n+1) .
$$

Theorem 3.1. Let $n, n_{1}, n_{2}$ be nonnegative integers with $n=1+n_{1}+n_{2}$. For $i=1,2$, let $\psi_{i}: N_{i} \rightarrow \mathbb{S}^{2 n_{i}+1} \subset \mathbb{C}^{n_{i}+1}$ be Legendrian isometric immersions of $n_{i}$-dimensional oriented Riemannian manifolds $\left(N_{i}, g_{i}\right)$. Suppose $\gamma=\left(\gamma_{1}, \gamma_{2}\right)$ : $I \rightarrow \mathbb{S}^{3} \subset \mathbb{C}^{2}$ is a Legendre curve, where $I$ is an interval in $\mathbb{R}$. The map

$$
\phi: I \times N_{1} \times N_{2} \rightarrow \mathbb{S}^{2 n+1} \subset \mathbb{C}^{n+1}=\mathbb{C}^{n_{1}+1} \times \mathbb{C}^{n_{2}+1}
$$

defined by

$$
\phi(s, p, q)=\left(\gamma_{1}(s) \psi_{1}(p), \gamma_{2}(s) \psi_{2}(q)\right)
$$

is a Legendrian immersion in $\mathbb{S}^{2 n+1}$ whose induced metric is

$$
\langle,\rangle=\left|\gamma^{\prime}\right|^{2} d s^{2}+\left|\gamma_{1}\right|^{2} g_{1}+\left|\gamma_{2}\right|^{2} g_{2}
$$

and whose Legendrian angle map is

$$
\beta_{\phi} \equiv n_{1} \pi+\beta_{\gamma}+n_{1} \arg \gamma_{1}+n_{2} \arg \gamma_{2}+\beta_{\psi_{1}}+\beta_{\psi_{2}} \bmod 2 \pi,
$$

where $\beta_{\gamma}, \beta_{\psi_{1}}$ and $\beta_{\psi_{2}}$ are the Legendre angle maps of $\gamma, \psi_{1}$ and $\psi_{2}$.

If $n_{1}, n_{2} \geq 2$, a Legendrian immersion $M^{n} \rightarrow \mathbb{S}^{2 n+1}$ is invariant under the action (3) of $\mathrm{SO}\left(n_{1}+1\right) \times \mathrm{SO}\left(n_{2}+1\right)$ if and only if it is locally of the form (4), where $\psi_{i}$ $(i=1,2)$ is the totally geodesic Legendrian embedding of $\mathbb{S}^{n_{i}}$ in $\mathbb{S}^{2 n_{i}+1}$ and $\gamma$ is some Legendre curve in $\mathbb{S}^{3}$. That is, such immersions are locally congruent to $\left(s, x_{1}, x_{2}\right) \mapsto\left(\gamma_{1}(s) x_{1}, \gamma_{2}(s) x_{2}\right)$, where $x_{i} \in \mathbb{S}^{n_{i}}$.

Note that Legendrian immersions of the form (4) have singularities at the points $(s, p, q) \in I \times N_{1} \times N_{2}$ where either $\gamma_{1}(s)=0$ or $\gamma_{2}(s)=0$.

Proof. If ' denotes differentiation with respect to $s$, and $v$ and $w$ are arbitrary tangent vectors to $N_{1}$ and $N_{2}$ respectively, it is clear that

$$
\begin{aligned}
\phi_{s} & =\phi_{*}\left(\partial_{s}, 0,0\right)=\left(\gamma_{1}^{\prime} \psi_{1}, \gamma_{2}^{\prime} \psi_{2}\right), \\
\phi_{*}(v) & :=\phi_{*}(0, v, 0)=\left(\gamma_{1} \psi_{1 *}(v), 0\right), \\
\phi_{*}(w) & :=\phi_{*}(0,0, w)=\left(0, \gamma_{2} \psi_{2 *}(w)\right) .
\end{aligned}
$$


(Recall that $g_{1}, g_{2}$ are the metrics on $N_{1}, N_{2}$ induced by $\psi_{1}, \psi_{2}$.) Because $\psi_{1}$ and $\psi_{2}$ are Legendrian immersions, we deduce from these equalities that the induced metric on $I \times N_{1} \times N_{2}$ by $\phi$ is $\left|\gamma^{\prime}\right|^{2} d s^{2}+\left|\gamma_{1}\right|^{2} g_{1}+\left|\gamma_{2}\right|^{2} g_{2}$. It follows that, $\gamma, \psi_{1}$ and $\psi_{2}$ being Legendrian, so is the immersion $\phi$.

To compute the Legendrian angle map $\beta_{\phi}$, let $\left\{e_{1}, \ldots, e_{n_{1}}\right\}$ and $\left\{e_{1}^{\prime}, \ldots, e_{n_{2}}^{\prime}\right\}$ be oriented local orthonormal frames on $N_{1}$ and $N_{2}$. Then the frame

$$
\left\{u_{1}, v_{1}, \ldots, v_{n_{1}}, w_{1}, \ldots, w_{n_{2}}\right\}
$$

defined by

$$
u_{1}=\left(\frac{\partial_{s}}{\left|\gamma^{\prime}\right|}, 0,0\right), \quad v_{j}=\left(0, \frac{e_{j}}{\left|\gamma_{1}\right|}, 0\right), \quad w_{k}=\left(0,0, \frac{e_{k}^{\prime}}{\left|\gamma_{2}\right|}\right)
$$

(with $1 \leq j \leq n_{1}, 1 \leq k \leq n_{2}$ ) is a local oriented orthonormal frame on $I \times N_{1} \times N_{2}$. Putting

$$
\begin{aligned}
\phi & =\gamma_{1}\left(\psi_{1}, 0\right)+\gamma_{2}\left(0, \psi_{2}\right), \\
\phi_{*}\left(u_{1}\right) & =\frac{\gamma_{1}^{\prime}}{\left|\gamma^{\prime}\right|}\left(\psi_{1}, 0\right)+\frac{\gamma_{2}^{\prime}}{\left|\gamma^{\prime}\right|}\left(0, \psi_{2}\right),
\end{aligned}
$$

we have

$$
\begin{aligned}
e^{i \beta_{\phi}}= & \operatorname{det}_{\mathbb{C}}\left\{\phi, \phi_{*}\left(u_{1}\right), \ldots, \phi_{*}\left(v_{j}\right), \ldots, \phi_{*}\left(w_{k}\right), \ldots\right\} \\
= & \frac{\gamma_{1}^{n_{1}} \gamma_{2}^{n_{2}}\left(\gamma_{1} \gamma_{2}^{\prime}-\gamma_{1}^{\prime} \gamma_{2}\right)}{\left|\gamma^{\prime}\right|\left|\gamma_{1}\right|^{n_{1}}\left|\gamma_{2}\right|^{n_{2}}} \\
& \quad \times \operatorname{det}_{\mathbb{C}}\left\{\left(\psi_{1}, 0\right),\left(0, \psi_{2}\right), \ldots,\left(\psi_{1 *}\left(e_{j}\right), 0\right), \ldots,\left(0, \psi_{2 *}\left(e_{k}^{\prime}\right)\right), \ldots\right\} .
\end{aligned}
$$

In this way we obtain

$$
e^{i \beta_{\phi}(s, p, q)}=(-1)^{n_{1}} e^{i\left(n_{1} \arg \gamma_{1}+n_{2} \arg \gamma_{2}\right)(s)} \frac{\left(\gamma_{1} \gamma_{2}^{\prime}-\gamma_{1}^{\prime} \gamma_{2}\right)(s)}{\left|\gamma^{\prime}(s)\right|} \operatorname{det}_{\mathbb{C}} A_{1}(p) \operatorname{det}_{\mathbb{C}} A_{2}(q),
$$

where $A_{1}$ and $A_{2}$ are the matrices

$$
\begin{aligned}
& A_{1}=\left\{\psi_{1}, \psi_{1 *}\left(e_{1}\right), \ldots, \psi_{1 *}\left(e_{n_{1}}\right)\right\}, \\
& A_{2}=\left\{\psi_{2}, \psi_{2 *}\left(e_{1}^{\prime}\right), \ldots, \psi_{2 *}\left(e_{n_{2}}^{\prime}\right)\right\} .
\end{aligned}
$$

Taking into account the definition of the Legendrian angle map given in Section 2, we finally arrive at

$$
e^{i \beta_{\phi}(s, p, q)}=(-1)^{n_{1}} e^{i\left(\beta_{\gamma}+n_{1} \arg \gamma_{1}+n_{2} \arg \gamma_{2}\right)(s)} e^{i \beta_{\psi_{1}}(p)} e^{i \beta_{\psi_{2}}(q)} .
$$

This proves the first part of the result. 
Conversely, let $\psi: M^{n} \rightarrow \mathbb{S}^{2 n+1} \subset \mathbb{C}^{n+1}$ be a Legendrian immersion that is invariant under the action (3) of $\mathrm{SO}\left(n_{1}+1\right) \times \mathrm{SO}\left(n_{2}+1\right)$. Let $p$ be any point of $M$ and set $z=\left(z_{1}, \ldots, z_{n+1}\right)=\psi(p)$. By the invariance assumption, for any matrix $X=\left(X_{1}, X_{2}\right)$ in the Lie algebra of $\mathrm{SO}\left(n_{1}+1\right) \times \mathrm{SO}\left(n_{2}+1\right)$, the curve $t \mapsto z e^{t \hat{X}}$ given by

$$
\hat{X}=\left(\begin{array}{l|l}
X_{1} & \\
\hline & X_{2}
\end{array}\right)
$$

lies in the submanifold. Thus its tangent vector at $t=0$ satisfies

$$
z \hat{X} \in \psi_{*}\left(T_{p} M\right) \text {. }
$$

Since $\psi$ is a Legendrian immersion, this implies that

$$
\operatorname{Im}\left(z \hat{X} \hat{Y} \bar{z}^{t}\right)=0
$$

for any matrices $X=\left(X_{1}, X_{2}\right), Y=\left(Y_{1}, Y_{2}\right)$ in the Lie algebra of $\mathrm{SO}\left(n_{1}+1\right) \times$ $\mathrm{SO}\left(n_{2}+1\right)$. As $n_{1}+1 \geq 3$ and $n_{2}+1 \geq 3$, it is easy to see from the last equation that $\operatorname{Re}\left(z_{1}, \ldots, z_{n_{1}+1}\right)$ and $\operatorname{Im}\left(z_{1}, \ldots, z_{n_{1}+1}\right)$ are linearly dependent, and so are $\operatorname{Re}\left(z_{n_{1}+2}, \ldots, z_{n+1}\right)$ and $\operatorname{Im}\left(z_{n_{1}+2}, \ldots, z_{n+1}\right)$. But $\mathrm{SO}\left(n_{1}+1\right)$ acts transitively on $\mathbb{S}^{n_{1}}$ and $\mathrm{SO}\left(n_{2}+1\right)$ acts transitively on $\mathbb{S}^{n_{2}}$; hence $z$ is in the orbit (under the action of $\mathrm{SO}\left(n_{1}+1\right) \times \mathrm{SO}\left(n_{2}+1\right)$ described above) of a point of the form

$$
\left(z_{1}^{0}, 0, \ldots, 0, z_{n_{1}+2}^{0}, 0, \ldots, 0\right)
$$

with

$$
\left|z_{1}^{0}\right|^{2}=\sum_{i=1}^{n_{1}+1}\left|z_{i}\right|^{2} \text { and } \quad\left|z_{n_{1}+2}^{0}\right|^{2}=\sum_{j=n_{1}+2}^{n+1}\left|z_{j}\right|^{2} .
$$

This implies that locally $\psi$ is the orbit under the action of $\mathrm{SO}\left(n_{1}+1\right) \times \mathrm{SO}\left(n_{2}+1\right)$ of a curve $\gamma$ in $\mathbb{C}^{2} \equiv \mathbb{C}^{n} \cap\left\{z_{2}=\cdots=z_{n_{1}+1}=z_{n_{1}+3}=\cdots=z_{n+1}=0\right\}$. Therefore $M$ is locally $I \times \mathbb{S}^{n_{1}} \times \mathbb{S}^{n_{2}}$, with $I$ an interval in $\mathbb{R}$. Moreover, $\psi$ is given by

$$
\psi(s, x, y)=\left(\gamma_{1}(s) x, \gamma_{2}(s) y\right),
$$

where $\gamma=\left(\gamma_{1}, \gamma_{2}\right)$ must be a Legendre curve in $\mathbb{S}^{3} \subset \mathbb{C}^{2}$. Finally, as $\psi$ is a Legendrian submanifold, the result follows using the first part of this theorem.

In the next result we make use of the method described in Theorem 3.1 to obtain new minimal and C-minimal Legendrian immersions, which will provide (projecting via the Hopf fibration) new nontrivial minimal and H-minimal immersions in $\mathbb{C} \mathbb{P}^{n}$. 
Corollary 3.2. Let $\psi_{i}: N_{i} \rightarrow \mathbb{S}^{2 n_{i}+1}, i=1,2$, be $C$-minimal Legendrian immersions of $n_{i}$-dimensional oriented Riemannian manifolds $N_{i}, i=1,2$, and let $\gamma=\left(\gamma_{1}, \gamma_{2}\right): I \rightarrow \mathbb{S}^{3} \subset \mathbb{C}^{2}$ be a Legendre curve. As before, set $n=n_{1}+n_{2}+1$. Then the Legendrian immersion $\phi: I \times N_{1} \times N_{2} \rightarrow \mathbb{S}^{2 n+1}$ of Theorem 3.1, given by

$$
\phi(t, p, q)=\left(\gamma_{1}(t) \psi_{1}(p), \gamma_{2}(t) \psi_{2}(q)\right)
$$

is $C$-minimal if and only if there exist real constants $\lambda, \mu$ such that $\left(\gamma_{1}, \gamma_{2}\right)$ is a solution of the system of ordinary differential equations

$$
\left(\gamma_{1}^{\prime} \bar{\gamma}_{1}\right)(t)=-\left(\gamma_{2}^{\prime} \bar{\gamma}_{2}\right)(t)=-e^{i(\lambda+\mu t)} \bar{\gamma}_{1}(t)^{n_{1}+1} \bar{\gamma}_{2}(t)^{n_{2}+1}
$$

This Legendrian immersion $\phi$ is minimal if and only if $\psi_{1}$ and $\psi_{2}$ are minimal and there exists some $\lambda$ such that $\left(\gamma_{1}, \gamma_{2}\right)$ is a solution of the system (8) with $\mu=0$.

Remark. If we apply a rotation through $\theta$ to a Legendre curve $\gamma$ that is a solution of (8) with parameters $(\lambda, \mu)$, the new Legendre curve is a solution of the same equation with parameters $(\lambda-(n+1) \theta, \mu)$. The corresponding immersions given in Corollary 3.2 are related by $\tilde{\phi}=e^{i \theta} \phi$ and are therefore congruent. By choosing $\theta$ appropriately, then, we can assume that $\lambda=\pi$; that is, it suffices (up to congruence) to consider solutions of the one-parameter family of equations

$$
\left(\gamma_{j}^{\prime} \bar{\gamma}_{j}\right)(t)=(-1)^{j-1} i e^{i \mu t} \bar{\gamma}_{1}(t)^{n_{1}+1} \bar{\gamma}_{2}(t)^{n_{2}+1}, \quad \text { with } \mu \in \mathbb{R}, j=1,2 .
$$

Proof of Corollary 3.2. We know from Proposition 2.4 that $\phi$ is C-minimal if and only if $\Delta \beta_{\phi}=0$, where $\beta_{\phi}$ is given by (6). So we must compute the Laplacian of $\beta_{\phi}$. We use the orthonormal frame (7) and after a long but direct computation we obtain

$$
\Delta \beta_{\phi}=\frac{1}{\left|\gamma^{\prime}\right|^{2}}\left(\frac{\partial^{2} \beta_{\phi}}{\partial s^{2}}+\frac{\mathrm{d}}{\mathrm{d} s}\left(\log \frac{\left|\gamma_{1}\right|^{n_{1}}\left|\gamma_{2}\right|^{n_{2}}}{\left|\gamma^{\prime}\right|}\right) \frac{\partial \beta_{\phi}}{\partial s}\right)+\frac{\Delta_{1} \beta_{\psi_{1}}}{\left|\gamma_{1}\right|^{2}}+\frac{\Delta_{2} \beta_{\psi_{2}}}{\left|\gamma_{2}\right|^{2}},
$$

where the $\Delta_{i}$ are the Laplace operators in $\left(N_{i}, g_{i}\right)$.

The assumptions of the Corollary 3.2 imply that $\Delta_{1} \beta_{\psi_{1}}=\Delta_{2} \beta_{\psi_{2}}=0$ again by Proposition 2.4. So $\phi$ is C-minimal if and only if

$$
\frac{\partial^{2} \beta_{\phi}}{\partial s^{2}}+\frac{\mathrm{d}}{\mathrm{d} s}\left(\log \frac{\left|\gamma_{1}\right|^{n_{1}}\left|\gamma_{2}\right|^{n_{2}}}{\left|\gamma^{\prime}\right|}\right) \frac{\partial \beta_{\phi}}{\partial s}=0
$$

Since we want $\phi$ to be regular, we impose that $\gamma_{1}(0)$ and $\gamma_{2}(0)$ not vanish (see after statement of Theorem 3.1). Up to a reparametrization, we can assume that $\gamma$ satisfies $\left|\gamma^{\prime}(t)\right|=\left|\gamma_{1}(t)\right|^{n_{1}}\left|\gamma_{2}(t)\right|^{n_{2}}$. Thus (11) becomes

$$
\frac{\partial^{2} \beta_{\phi}}{\partial t^{2}}=0
$$


This means that $\beta_{\phi}(t, p, q)=f(p, q)+t g(p, q)$, for certain functions $f, g$ defined on $N_{1} \times N_{2}$. Using (6), we obtain that $g(p, q)$ is constant and that

$$
\left(\beta_{\gamma}+n_{1} \arg \gamma_{1}+n_{2} \arg \gamma_{2}\right)(t)=\lambda+\mu t, \quad \text { with } \lambda, \mu \in \mathbb{R} .
$$

The definition of the Legendrian angle $\beta_{\gamma}$ of $\gamma$ is given, in particular, by

$$
e^{i \beta_{\gamma}}=\frac{1}{\left|\gamma^{\prime}\right|}\left(\gamma_{1} \gamma_{2}^{\prime}-\gamma_{2} \gamma_{1}^{\prime}\right)
$$

Using this, it is easy to rewrite (12) as

$$
\gamma_{1}^{\prime} \bar{\gamma}_{1}=-\gamma_{2}^{\prime} \bar{\gamma}_{2}=-e^{i(\lambda+\mu t)} \bar{\gamma}_{1}^{n_{1}+1} \bar{\gamma}_{2}^{n_{2}+1},
$$

which is exactly (8).

Finally, by Proposition 2.2, $\phi$ is minimal if and only if $\beta_{\phi}$ is constant. This is equivalent to $\beta_{\psi_{1}}, \beta_{\psi_{2}}$ being constant (i.e., the $\psi_{i}$ are minimal, again by the same proposition) and $\beta_{\gamma}+n_{1} \arg \gamma_{1}+n_{2} \arg \gamma_{2}$ is constant. But this corresponds to the case $\mu=0$ in (12) and so to the case $\mu=0$ in (8).

It is difficult to describe the general solution of (9). However it is an exercise to check that for any $\delta \in(0, \pi / 2)$ the Legendre curve

$$
\gamma_{\delta}(t)=\left(c_{\delta} \exp \left(i s_{\delta}^{n_{1}+1} c_{\delta}^{n_{2}-1} t\right), s_{\delta} \exp \left(-i s_{\delta}^{n_{1}-1} c_{\delta}^{n_{2}+1} t\right)\right),
$$

satisfies (9) for $\mu=s_{\delta}^{n_{1}-1} c_{\delta}^{n_{2}-1}\left(\left(n_{1}+1\right) s_{\delta}^{2}-\left(n_{2}+1\right) c_{\delta}^{2}\right)$, where $c_{\delta}=\cos \delta$ and $s_{\delta}=\sin \delta$. This value of $\mu$ vanishes if and only if $\tan ^{2} \delta=\left(n_{2}+1\right) /\left(n_{1}+1\right)$. In this way we are able to obtain an explicit family of examples:

Corollary 3.3. Let $\psi_{i}: N_{i} \rightarrow \mathbb{S}^{2 n_{i}+1}$, for $i=1,2$, be C-minimal Legendrian immersions of $n_{i}$-dimensional Riemannian manifolds $N_{i}$, and let $n=n_{1}+n_{2}+1$. Given $\delta \in(0, \pi / 2)$, set $c_{\delta}=\cos \delta$ and $s_{\delta}=\sin \delta$. Then the map $\phi_{\delta}: \mathbb{R} \times N_{1} \times N_{2} \rightarrow$ $\mathbb{S}^{2 n+1}$ defined by

$$
\phi_{\delta}(t, p, q)=\left(c_{\delta} \exp \left(i s_{\delta}^{n_{1}+1} c_{\delta}^{n_{2}-1} t\right) \psi_{1}(p), s_{\delta} \exp \left(-i s_{\delta}^{n_{1}-1} c_{\delta}^{n_{2}+1} t\right) \psi_{2}(q)\right)
$$

is a C-minimal Legendrian immersion.

In particular, using minimal Legendrian immersions $\psi_{1}, \psi_{2}$ and the value $\delta=$ $\delta_{0}:=\arctan \sqrt{\left(n_{2}+1\right) /\left(n_{1}+1\right)}$, we obtain a minimal Legendrian immersion $\phi_{\delta_{0}}$ : $\mathbb{R} \times N_{1} \times N_{2} \rightarrow \mathbb{S}^{2 n+1}$.

Proof. We simply remark that we do not need the orientability assumption because, in the case at hand, the Legendrian immersions $\phi_{\delta}$ are easily seen to satisfy $\operatorname{div} J H=0$ and thus are C-minimal (see Definition 2.3).

To finish this section, we turn our attention to Equation (9) with $\mu=0$. We observe that this is exactly equation (6) in [Castro and Urbano 2004, Lemma 2] (in the notation of that paper, put $p=n_{1}$ and $q=n_{2}$ ). If we choose the initial 
conditions $\gamma(0)=(\cos \theta, \sin \theta)$, with $\theta \in(0, \pi / 2)$, we can make use of the study made in that reference.

Lemma 3.4. Let $\gamma_{\theta}=\left(\gamma_{1}, \gamma_{2}\right): I \subset \mathbb{R} \rightarrow \mathbb{S}^{3}$ be the unique curve solution of

$$
\gamma_{j}^{\prime} \bar{\gamma}_{j}=(-1)^{j-1} i \bar{\gamma}_{1}^{n_{1}+1} \bar{\gamma}_{2}^{n_{2}+1}, j=1,2,
$$

satisfying the real initial conditions $\gamma_{\theta}(0)=(\cos \theta, \sin \theta), \theta \in(0, \pi / 2)$.

(1) $\operatorname{Re}\left(\gamma_{1}^{n_{1}+1} \gamma_{2}^{n_{2}+1}\right)=\cos ^{n_{1}+1} \theta \sin ^{n_{2}+1} \theta$.

(2) For $j=1,2$ and any $t \in I$, we have $\bar{\gamma}_{j}(t)=\gamma_{j}(-t)$.

(3) The functions $\left|\gamma_{1}\right|$ and $\left|\gamma_{2}\right|$ are periodic with the same period $T=T(\theta)$, and $\gamma_{\theta}$ is a closed curve if and only if

$$
\theta \in(0, \pi / 2) \quad \text { and } \quad \frac{\cos ^{n_{1}+1} \theta \sin ^{n_{2}+1} \theta}{2 \pi}\left(\int_{0}^{T} \frac{d t}{\left|\gamma_{1}\right|^{2}(t)}, \int_{0}^{T} \frac{d t}{\left|\gamma_{2}\right|^{2}(t)}\right) \in \mathbb{Q}^{2} .
$$

(4) If $\theta$ takes the value $\delta_{0}=\arctan \sqrt{\left(n_{2}+1\right) /\left(n_{1}+1\right)}$ from Corollary 3.3, we recover the curve of Equation (13), with $\delta=\delta_{0}$.

Proof. (1) and (2) follow directly from parts 2 and 3 of [Castro and Urbano 2004, Lemma 2]. To prove (3) we set $f(\theta)=\cos ^{2\left(n_{1}+1\right)} \theta \sin ^{2\left(n_{2}+1\right)} \theta$, for $\theta \in(0, \pi / 2)$. It is easy to prove that $f(\theta) \leq\left(n_{1}+1\right)^{n_{1}+1}\left(n_{2}+1\right)^{n_{2}+1} /(n+1)^{n+1}$ and the equality holds if and only if $\theta=\delta_{0}$. Using this in parts 4 and 5 of [Castro and Urbano 2004, Lemma 2] completes the proof.

\section{H-minimal Lagrangian submanifolds in complex projective space}

In Section 2 we explained that we can construct (minimal, H-minimal) Lagrangian submanifolds in $\mathbb{C P}^{n}$ by projecting (minimal, C-minimal) Legendrian submanifolds in $\mathbb{S}^{2 n+1}$ by the Hopf fibration $\Pi: \mathbb{S}^{2 n+1} \rightarrow \mathbb{C} \mathbb{P}^{n}$ (Proposition 2.4). The aim of this section is to analyze the Lagrangian immersions in $\mathbb{C P}^{n}$ that we obtain just by projecting the Legendrian ones deduced in Section 3.

First we mention that if $n_{2}=0$ in Theorem 3.1, projection by the Hopf fibration $\Pi$ yields Examples 1 of [Castro et al. 2001]. In this sense, the construction given in Theorem 3.1 can be considered as a generalization of the family introduced in that reference. Some applications of our construction of Theorem 3.1 when $n=3$ have been used recently in [Montealegre and Vrancken 2006] to the study of minimal Lagrangian submanifolds in $\mathbb{C} \mathbb{P}^{3}$.

The Legendrian immersions described in Corollary 3.2 provide new examples of Lagrangian $\mathrm{H}$-minimal immersions in $\mathbb{C P}^{n}$ when we project them via $\Pi$. If we consider the case $n_{2}=0$ (so $n_{1}=n-1$ ) in the minimal case of Corollary 3.2, we recover (by projecting via the Hopf fibration $\Pi$ ) the minimal Lagrangian 
submanifolds of $\mathbb{C} \mathbb{P}^{n}$ described in [Castro et al. 2002, Proposition 6], although we used there a unit speed parametrization for $\gamma$.

We write in more detail what we obtain with this procedure if we consider the special case coming from Corollary 3.3.

Corollary 4.1. Let $\psi_{i}: N_{i} \rightarrow \mathbb{S}^{2 n_{i}+1}$, for $i=1,2$, be $C$-minimal Legendrian immersions of $n_{i}$-dimensional Riemannian manifolds $N_{i}$, and let $n=n_{1}+n_{2}+1$. Suppose $\delta \in(0, \pi / 2)$. Then the map $\Phi_{\delta}: \mathbb{S}^{1} \times N_{1} \times N_{2} \rightarrow \mathbb{C P}^{n}$ given by

$$
\Phi_{\delta}\left(e^{i s}, p, q\right)=\left[\left(\cos \delta \exp \left(i s \sin ^{2} \delta\right) \psi_{1}(p), \sin \delta \exp \left(-i s \cos ^{2} \delta\right) \psi_{2}(q)\right)\right]
$$

is an H-minimal Lagrangian immersion. $\Phi_{\delta}$ is minimal if and only if $\psi_{1}$ and $\psi_{2}$ are minimal and $\tan ^{2} \delta=\left(n_{2}+1\right) /\left(n_{1}+1\right)$. (Recall that the brackets denote the image under П.)

Proof. We consider the C-minimal Legendrian immersions

$$
\phi_{\delta}: \mathbb{R} \times N_{1} \times N_{2} \rightarrow \mathbb{S}^{2 n+1}
$$

given in Corollary 3.3. Projecting via the Hopf fibration $\Pi: \mathbb{S}^{2 n+1} \rightarrow \mathbb{C P}^{n}$ and using Proposition 2.4 we conclude that

$$
\Pi \circ \phi_{\delta}: \mathbb{R} \times N_{1} \times N_{2} \rightarrow \mathbb{C P}^{n}
$$

is a one-parameter family of H-minimal Lagrangian immersions. We study when $\Pi \circ \phi_{\delta}$ is periodic in its first variable. It is easy to see that there exists $A>0$ such that $\left(\Pi \circ \phi_{\delta}\right)(t+A, p, q)=\left(\Pi \circ \phi_{\delta}\right)(t, p, q), \forall(t, p, q) \in \mathbb{R} \times N_{1} \times N_{2}$ if and only if there exists $\theta \in \mathbb{R}$ satisfying

$$
\exp \left(i s_{\delta}^{n_{1}+1} c_{\delta}^{n_{2}-1} A\right)=e^{i \theta}=\exp \left(-i s_{\delta}^{n_{1}-1} c_{\delta}^{n_{2}+1} A\right) .
$$

We deduce that the smallest period $A$ must equal $A=2 \pi /\left(s_{\delta}^{n_{1}-1} c_{\delta}^{n_{2}-1}\right)$. Applying the change of variables

$$
s \mapsto t=s /\left(s_{\delta}^{n_{1}-1} c_{\delta}^{n_{2}-1}\right)
$$

for $s \in[0,2 \pi]$, the equation for the Legendre curve $\gamma_{\delta}$ of (13) becomes

$$
\gamma_{\delta}(s)=\left(c_{\delta} \exp \left(i s_{\delta}^{2} s\right), s_{\delta} \exp \left(-i c_{\delta}^{2} s\right)\right), s \in[0,2 \pi],
$$

which leads to the expression of $\Phi_{\delta}$.

We conclude the proof by observing that $\Pi \circ \phi_{\delta}$ is minimal if and only if $\phi_{\delta}$ is minimal (see Section 2) and using Corollary 3.3 again.

We get $\mathrm{H}$-minimal Lagrangian embeddings as a particular case: 
Corollary 4.2. Let $\delta \in(0, \pi / 2)$ and $n=n_{1}+n_{2}+1$. The immersion $\Phi_{\delta}$ of Corollary 3.3, where $\psi_{i}$, for $i=1,2$, is the totally geodesic Legendrian embedding of $\mathbb{S}^{n_{i}}$ into $\mathbb{S}^{2 n_{i}+1}$, gives rise to an H-minimal Lagrangian embedding

$$
\overline{\left(e^{i s}, x, y\right)} \mapsto\left[\left(\cos \delta \exp \left(i s \sin ^{2} \delta\right) x, \sin \delta \exp \left(-i s \cos ^{2} \delta\right) y\right)\right]
$$

of the quotient $\left(\mathbb{S}^{1} \times \mathbb{S}^{n_{1}} \times \mathbb{S}^{n_{2}}\right) /\left(\mathbb{Z}_{2} \times \mathbb{Z}_{2}\right)$ into $\mathbb{C} \mathbb{P}^{n}$, the action of $\mathbb{Z}_{2} \times \mathbb{Z}_{2}$ being generated by the involutions $\left(e^{i s}, x, y\right) \mapsto\left(-e^{i s},-x, y\right),\left(e^{i s}, x, y\right) \mapsto\left(-e^{i s}, x,-y\right)$.

Proof. Consider the H-minimal Lagrangian immersion $\Phi_{\delta}: \mathbb{S}^{1} \times \mathbb{S}^{n_{1}} \times \mathbb{S}^{n_{2}} \rightarrow \mathbb{C} \mathbb{P}^{n}$ defined by

$$
\Phi_{\delta}\left(e^{i s}, x, y\right)=\left[\left(\cos \delta \exp \left(i \sin ^{2} \delta s\right) x, \sin \delta \exp \left(-i \cos ^{2} \delta s\right) y\right)\right] .
$$

Take $\left(e^{i s}, x, y\right),\left(e^{i \hat{s}}, \hat{x}, \hat{y}\right) \in \mathbb{S}^{1} \times \mathbb{S}^{n_{1}} \times \mathbb{S}^{n_{2}}$. Then $\Phi_{\delta}\left(e^{i s}, x, y\right)=\Phi_{\delta}\left(e^{i \hat{s}}, \hat{x}, \hat{y}\right)$ if and only if there exists $\theta \in \mathbb{R}$ such that

$$
\hat{x}=\exp \left(i\left(\theta+\sin ^{2} \delta(s-\hat{s})\right)\right) x, \quad \hat{y}=\exp \left(i\left(\theta-\cos ^{2} \delta(s-\hat{s})\right)\right) y .
$$

Since some coordinate of $x \in \mathbb{S}^{n_{1}}$ and $y \in \mathbb{S}^{n_{2}}$ is nonzero, we deduce that

$$
\begin{aligned}
& \epsilon_{1}:=\exp \left(i\left(\theta+\sin ^{2} \delta(s-\hat{s})\right)\right)= \pm 1, \\
& \epsilon_{2}:=\exp \left(i\left(\theta-\cos ^{2} \delta(s-\hat{s})\right)\right)= \pm 1 .
\end{aligned}
$$

We distinguish two cases:

(i) $\epsilon_{1}=\epsilon_{2}$ : From (16) we get $e^{i \hat{s}}=e^{i s}$; using (15) we obtain $\hat{x}=x, \hat{y}=y$ if $\epsilon_{1}=\epsilon_{2}=1$ or $\hat{x}=-x, \hat{y}=-y$ if $\epsilon_{1}=\epsilon_{2}=-1$. In either case $\left(e^{i \hat{s}}, \hat{x}, \hat{y}\right)$ and $\left(e^{i s}, x, y\right)$ are equivalent under the $\mathbb{Z}_{2} \times \mathbb{Z}_{2}$ action.

(ii) $\epsilon_{1}=-\epsilon_{2}$ : From (16) we get $e^{i \hat{s}}=-e^{i s}$ and using (15) we obtain that either $\hat{x}=x$ and $\hat{y}=-y$ or $\hat{x}=-x$ and $\hat{y}=y$. Again we see that $\left(e^{i \hat{s}}, \hat{x}, \hat{y}\right)$ and $\left(e^{i s}, x, y\right)$ are equivalent under the $\mathbb{Z}_{2} \times \mathbb{Z}_{2}$ action.

If $\tan ^{2} \delta=\left(n_{2}+1\right) /\left(n_{1}+1\right)$ the minimal Lagrangian embedding of Corollary 4.2 admits as a special case $\left(n_{2}=0\right)$ the example $\left(\mathbb{S}^{1} \times \mathbb{S}^{n-1}\right) / \mathbb{Z}_{2} \rightarrow \mathbb{C} \mathbb{P}^{n}$ studied in [Naitoh 1981].

Remark 4.3. As can easily be checked, the action of $\mathbb{Z}_{2} \times \mathbb{Z}_{2}$ on $\mathbb{S}^{1} \times \mathbb{S}^{n_{1}} \times \mathbb{S}^{n_{2}}$ preserves orientation (and hence the quotient is an orientable manifold) if and only if both $n_{1}$ and $n_{2}$ are odd.

To conclude this section, we use the information given by Lemma 3.4 on the solutions of equation (9) with $\mu=0$.

Assume $\theta \in(0, \pi / 2)$ and let $\gamma_{\theta}$ be the only solution of (14) satisfying $\gamma_{\theta}(0)=$ $(\cos \theta, \sin \theta)$. Consider the C-minimal Legendrian immersions

$$
\phi_{\theta}: I \times N_{1} \times N_{2} \rightarrow \mathbb{S}^{2 n+1}
$$


constructed with $\gamma_{\theta}$. Projecting by the Hopf fibration $\Pi: \mathbb{S}^{2 n+1} \rightarrow \mathbb{C P}^{n}$ and using Proposition 2.4 we obtain a one-parameter family

$$
\Pi \circ \phi_{\theta}: I \times N_{1} \times N_{2} \rightarrow \mathbb{C P}^{n}
$$

of H-minimal Lagrangian immersions.

Lemma 3.4(3) tells us when $\gamma_{\theta}$ is a closed curve, but now we want to find when $\Pi \circ \phi_{\theta}$ is periodic of period $T$, say, in its first variable. Write $\gamma_{\theta}=\left(\rho_{1} e^{i v_{1}}, \rho_{2} e^{i \nu_{2}}\right)$; then $\rho_{i}(t+T)=\rho_{i}(t)$ for $i=1,2$. It is not hard to deduce that there exists $A>0$ such that $\left(\Pi \circ \phi_{\theta}\right)(t+A, p, q)=\left(\Pi \circ \phi_{\theta}\right)(t, p, q)$ if and only if there exist $v \in \mathbb{R}$ and $m \in \mathbb{Z}$ satisfying

$$
e^{i v_{j}(t+m T)}=e^{i v} e^{i v_{j}(t)}, \quad j=1,2
$$

(and then $A=m T$ ). From (14) we can deduce that

$$
\rho_{j}^{2} v_{j}^{\prime}=(-1)^{j-1} c_{\theta}^{n_{1}+1} s_{\theta}^{n_{2}+1}, \quad j=1,2 .
$$

Then it is easy to check that $v_{j}(t+m T)=v_{j}(t)+m v_{j}(T), j=1,2$, and (17) is equivalent to $e^{i m v_{j}(T)}=e^{i v}, j=1,2$. This means that $\left(\nu_{2}(T)-v_{1}(T)\right) / 2 \pi$ must be a rational number. In view of (18), this implies that $\theta$ lies in

$$
\Gamma:=\left\{\alpha \in\left(0, \frac{\pi}{2}\right): \frac{\cos ^{n_{1}+1} \alpha \sin ^{n_{2}+1} \alpha}{2 \pi} \int_{0}^{T} \frac{d t}{\left|\gamma_{1}\right|^{2}(t)\left|\gamma_{2}\right|^{2}(t)} \in \mathbb{Q}\right\} .
$$

Hence:

Corollary 4.4. For $\theta \in \Gamma$ and fixed $C$-minimal Legendrian immersions $\psi_{i}: N_{i} \rightarrow$ $\mathbb{S}^{2 n_{i}+1}, i=1,2$, we obtain from $\phi_{\theta}$ a one-parameter family of $H$-minimal Lagrangian immersions

$$
\Phi_{\theta}: \mathbb{S}^{1} \times N_{1} \times N_{2} \rightarrow \mathbb{C P}^{n}, \quad n=n_{1}+n_{2}+1, \quad \theta \in \Gamma .
$$

In particular, $\Phi_{\theta}$ is minimal if and only if $\psi_{1}$ and $\psi_{2}$ are.

\section{H-minimal Lagrangian cones in complex Euclidean space}

Given a Legendrian immersion $\phi: M^{n} \rightarrow \mathbb{S}^{2 n+1}$, the cone with link $\phi$ in $\mathbb{C}^{n+1}$ is the map $C(\phi): \mathbb{R} \times M^{n} \rightarrow \mathbb{C}^{n+1}$ given by

$$
(s, p) \mapsto s \phi(p) .
$$

$C(\phi)$ is a Lagrangian immersion with singularities at $s=0$.

M. Haskins [2004b; 2004a] has studied in depth special Lagrangian cones using the fact that $\phi$ is minimal if and only if $C(\phi)$ is minimal. Following a reasoning similar to Haskin's, a straightforward computation leads to the next result. 
Proposition 5.1. Let $\phi: M^{n} \rightarrow \mathbb{S}^{2 n+1}$ be a Legendrian immersion of an oriented manifold $M$ and $C(\phi): \mathbb{R} \times M \rightarrow \mathbb{C}^{n+1}$ the cone with link $\phi$. Then $\phi$ is $C$-minimal if and only if $C(\phi)$ is H-minimal.

Thanks to Proposition 5.1 we have a fruitful and simple construction method for examples of $\mathrm{H}$-minimal Lagrangian cones in $\mathbb{C}^{n+1}$ using the $\mathrm{C}$-minimal Legendrian immersions described in Section 3.

\section{The complex hyperbolic case}

In this section we summarize the analogous results when the ambient space is complex hyperbolic space. We omit proofs.

Let $\mathbb{C}_{1}^{n+1}$ be complex Euclidean space $\mathbb{C}^{n+1}$ endowed with the indefinite metric $\langle\rangle=,\operatorname{Re}($,$) , where$

$$
(z, w)=\sum_{i=1}^{n} z_{i} \bar{w}_{i}-z_{n+1} \bar{w}_{n+1}
$$

for $z, w \in \mathbb{C}^{n+1}$, here $\bar{z}$ stands for the conjugate of $z$. The Liouville 1-form is given by $\Lambda_{z}(v)=\langle v, J z\rangle$, for all $z \in \mathbb{C}^{n+1}$ and all $v \in T_{z} \mathbb{C}^{n+1}$, and the Kähler 2-form is $\omega=d \Lambda / 2$. We denote by $\mathbb{Q}_{1}^{2 n+1}$ the anti-de Sitter space, defined as the hypersurface of $\mathbb{C}_{1}^{n+1}$ given by

$$
\mathbb{W}_{1}^{2 n+1}=\left\{z \in \mathbb{C}^{n+1} /(z, z)=-1\right\},
$$

and by $\Pi: \mathbb{U}_{1}^{2 n+1} \rightarrow \mathbb{C} \mathbb{H}^{n}, \Pi(z)=[z]$, the Hopf fibration of $\mathbb{U}_{1}^{2 n+1}$ onto complex hyperbolic space $\mathbb{C} \mathbb{H}^{n}$. The metric, complex structure and Kähler two-form in $\mathbb{C} \mathbb{H}^{n}$ are written $\langle\rangle,$,$J and \omega$. This metric has constant holomorphic sectional curvature -4 . We also denote by $\Lambda$ the restriction to $\mathbb{H}_{1}^{2 n+1}$ of the Liouville 1 -form of $\mathbb{C}_{1}^{n+1}$. Thus $\Lambda$ is the contact 1-form of the canonical (indefinite) Sasakian structure on the anti-de Sitter space $\mathbb{H}_{1}^{2 n+1}$. An immersion $\phi: M^{n} \rightarrow \mathbb{U}_{1}^{2 n+1}$ of an $n$-dimensional manifold $M$ is said to be Legendrian if $\phi^{*} \Lambda \equiv 0$. So $\phi$ is isotropic in $\mathbb{C}_{1}^{n+1}$, that is, $\phi^{*} \omega \equiv 0$. In particular, the normal bundle $T^{\perp} M$ has the decomposition $J(T M) \oplus \operatorname{span}\{J \phi\}$. This means that $\phi$ is horizontal with respect to the Hopf fibration $\Pi: \mathbb{M}_{1}^{2 n+1} \rightarrow \mathbb{C} \mathbb{M}^{n}$, and hence $\Phi=\Pi \circ \phi: M^{n} \rightarrow \mathbb{C}^{n}$ is a Lagrangian immersion and the induced metrics on $M^{n}$ by $\phi$ and $\Phi$ are the same.

It is easy to check that $J \phi$ is a totally geodesic normal vector field, so the second fundamental forms of $\phi$ and $\Phi$ are related by

$$
\Pi_{*}\left(\sigma_{\phi}(v, w)\right)=\sigma_{\Phi}\left(\Pi_{*} v, \Pi_{*} w\right) \text { for all } v, w \in T M .
$$

Thus the mean curvature vector $H$ of $\phi$ satisfies $\langle H, J \phi\rangle=0$. In particular, $\phi$ : $M^{n} \rightarrow \mathbb{M}_{1}^{2 n+1}$ is minimal if and only if $\Phi=\Pi \circ \phi: M^{n} \rightarrow \mathbb{C}^{n}$ is minimal. 
In this way, we can construct (minimal) Lagrangian submanifolds in $\mathbb{C} \mathbb{H}^{n}$ by projecting (minimal) Legendrian manifolds in $\mathbb{H}_{1}^{2 n+1}$ via the Hopf fibration $\Pi$.

Let $\Omega$ be the complex $n$-form on $\mathbb{\boxplus}_{1}^{2 n+1}$ given by

$$
\Omega_{z}\left(v_{1}, \ldots, v_{n}\right)=\operatorname{det}_{\mathbb{C}}\left\{z, v_{1}, \ldots, v_{n}\right\} .
$$

If $\phi: M^{n} \rightarrow \mathbb{H}_{1}^{2 n+1}$ is a Legendrian immersion of a manifold $M$, then $\phi^{*} \Omega$ is a complex $n$-form on $M$. In the following result we analyze this $n$-form $\phi^{*} \Omega$.

Lemma 6.1. If $\phi: M^{n} \rightarrow \mathbb{R}_{1}^{2 n+1}$ is a Legendrian immersion of a manifold $M$, then

$$
\nabla\left(\phi^{*} \Omega\right)=\alpha_{H} \otimes \phi^{*} \Omega,
$$

where $\alpha_{H}$ is the one-form on $M$ defined by $\alpha_{H}(v)=n i\langle H, J v\rangle$ and $H$ is the mean curvature vector of $\phi$. Consequently, $M$ is orientable if $\phi$ is minimal.

Suppose that our Legendrian submanifold $M$ is oriented. Consider the well defined map $\beta: M^{n} \rightarrow \mathbb{R} / 2 \pi \mathbb{Z}$ given by

$$
e^{i \beta(p)}=\left(\phi^{*} \Omega\right)_{p}\left(e_{1}, \ldots, e_{n}\right)
$$

where $\left\{e_{1}, \ldots, e_{n}\right\}$ is an oriented orthonormal frame in $T_{p} M$. We will call $\beta$ the Legendrian angle map of $\phi$. As a consequence of (19) we obtain

$$
J \nabla \beta=n H,
$$

and so we deduce:

Proposition 6.2. Let $\phi: M^{n} \rightarrow \mathbb{Q}_{1}^{2 n+1}$ be a Legendrian immersion of an oriented manifold $M$. Then $\phi$ is minimal if and only if the Legendrian angle map $\beta$ of $\phi$ is constant.

In this context we can also consider contact minimal (or briefly $C$-minimal) Legendrian submanifolds of $\mathbb{H}_{1}^{2 n+1}$ as critical points of the volume functional for compactly supported variations with variational vector field a (normal) contact field $\xi_{f}=J \nabla f-2 f J \phi$, where $f$ lies in $C_{0}^{\infty}(M)$ and $\nabla f$ is the gradient of $f$ respect to the induced metric. Such fields are also characterized by the equation $\operatorname{div} J H=0$, and we have a counterpart to Proposition 2.4:

Proposition 6.3. Let $\phi: M^{n} \rightarrow \mathbb{H}_{1}^{2 n+1}$ be a Legendrian immersion of a Riemannian manifold $M$.

(1) If $M$ is oriented, $\phi$ is $C$-minimal if and only if the Legendrian angle $\beta$ of $\phi$ is a harmonic map.

(2) $\phi$ is $C$-minimal if and only if $\Phi=\Pi \circ \phi: M^{n} \rightarrow \mathbb{C} \mathbb{H}^{n}$ is $H$-minimal. 
The identity component of the indefinite special orthogonal group will be denoted by $\mathrm{SO}_{0}^{1}(m)$. So $\mathrm{SO}\left(n_{1}+1\right) \times \mathrm{SO}_{0}^{1}\left(n_{2}+1\right)$ acts on $\mathbb{H}_{1}^{2 n+1} \subset \mathbb{C}^{n+1}$, where $n=n_{1}+n_{2}+1$, as a subgroup of isometries:

$$
\left(A_{1}, A_{2}\right) \in \mathrm{SO}\left(n_{1}+1\right) \times \mathrm{SO}_{0}^{1}\left(n_{2}+1\right) \mapsto\left(\begin{array}{l|l}
A_{1} \mid & \\
\hline & A_{2}
\end{array}\right) \in \mathrm{SO}_{0}^{1}(n+1) .
$$

We now state the main results of Section 3 adapted to this context. We denote by $\mathbb{R} \mathbb{T}^{n}=\left\{\left(y_{1}, \ldots, y_{n+1}\right) \in \mathbb{R}^{n+1}: \sum_{i=1}^{n} y_{i}^{2}-y_{n+1}^{2}=-1, y_{n+1}>0\right\}$ the real hyperbolic space of dimension $n$.

Theorem 6.4. Let $n, n_{1}, n_{2}$ be nonnegative integers with $n=1+n_{1}+n_{2}$. Let $\psi_{1}$ : $N_{1} \rightarrow \mathbb{S}^{2 n_{1}+1} \subset \mathbb{C}^{n_{1}+1}$ and $\psi_{2}: N_{2} \rightarrow \mathbb{H}_{1}^{2 n_{2}+1} \subset \mathbb{C}^{n_{2}+1}$ be Legendrian immersions of $n_{i}$-dimensional oriented Riemannian manifolds $\left(N_{i}, g_{i}\right)$. Suppose $\gamma=\left(\gamma_{1}, \gamma_{2}\right)$ : $I \rightarrow \mathbb{M}_{1}^{3} \subset \mathbb{C}^{2}$ is a Legendre curve. The map

$$
\phi: I \times N_{1} \times N_{2} \rightarrow \mathbb{H}_{1}^{2 n+1} \subset \mathbb{C}^{n+1}=\mathbb{C}^{n_{1}+1} \times \mathbb{C}^{n_{2}+1}
$$

defined by

$$
\phi(s, p, q)=\left(\gamma_{1}(s) \psi_{1}(p), \gamma_{2}(s) \psi_{2}(q)\right)
$$

is a Legendrian immersion in $\mathbb{\boxplus}_{1}^{2 n+1}$ whose induced metric is

$$
\langle,\rangle=\left|\gamma^{\prime}\right|^{2} d s^{2}+\left|\gamma_{1}\right|^{2} g_{1}+\left|\gamma_{2}\right|^{2} g_{2}
$$

and whose Legendrian angle map is

$$
\beta_{\phi} \equiv n_{1} \pi+\beta_{\gamma}+n_{1} \arg \gamma_{1}+n_{2} \arg \gamma_{2}+\beta_{\psi_{1}}+\beta_{\psi_{2}} \bmod 2 \pi,
$$

where $\beta_{\gamma}, \beta_{\psi_{1}}$ and $\beta_{\psi_{2}}$ are the Legendre angle maps of $\gamma, \psi_{1}$ and $\psi_{2}$.

If $n_{1}, n_{2} \geq 2$, a Legendrian immersion $M^{n} \rightarrow \mathbb{M}_{1}^{2 n+1}$ is invariant under the action (20) of $\mathrm{SO}\left(n_{1}+1\right) \times \mathrm{SO}_{0}^{1}\left(n_{2}+1\right)$ if and only if it is locally of the form (21), where $\psi_{1}$ is the totally geodesic Legendrian embedding of $\mathbb{S}^{n_{1}}$ in $\mathbb{S}^{2 n_{1}+1}$ and $\psi_{2}$ is the totally geodesic Legendrian embedding of $\mathbb{R} \mathbb{H}^{n_{2}}$ in $\mathbb{H}_{1}^{2 n_{2}+1}$ That is, such immersions are locally congruent to $\phi(s, x, y)=\left(\gamma_{1}(s) x, \gamma_{2}(s) y\right)$, where $x \in \mathbb{S}^{n_{1}}, y \in \mathbb{R} \mathbb{H}^{n_{2}}$.

Remark. If $n_{2}=0$ in the theorem, we recover Examples 2 of [Castro et al. 2001] by projection via the Hopf fibration $\Pi: \mathbb{M}_{1}^{2 n+1} \rightarrow \mathbb{C}^{n}$. When $n_{1}=0$ we obtain Examples 3.

Corollary 6.5. Let $\psi_{1}: N_{1} \rightarrow \mathbb{S}^{2 n_{1}+1} \subset \mathbb{C}^{n_{1}+1}$ and $\psi_{2}: N_{2} \rightarrow \mathbb{\boxplus}_{1}^{2 n_{2}+1} \subset \mathbb{C}^{n_{2}+1}$ be Cminimal Legendrian immersions of $n_{i}$-dimensional oriented Riemannian manifolds $N_{i}, i=1,2$, and let $\gamma=\left(\gamma_{1}, \gamma_{2}\right): I \rightarrow \mathbb{M}_{1}^{3} \subset \mathbb{C}^{2}$ be a Legendre curve. As before, set $n=n_{1}+n_{2}+1$. Then the Legendrian immersion $\phi: I \times N_{1} \times N_{2} \rightarrow \mathbb{H}_{1}^{2 n+1}$ of 
Theorem 6.4, given by

$$
\phi(t, p, q)=\left(\gamma_{1}(t) \psi_{1}(p), \gamma_{2}(t) \psi_{2}(q)\right),
$$

is $C$-minimal if and only if, up to congruences, there exists a real constant $\mu$ such that $\left(\gamma_{1}, \gamma_{2}\right)$ is a solution of the system of ordinary differential equations

$$
\left(\gamma_{1}^{\prime} \bar{\gamma}_{1}\right)(t)=\left(\gamma_{2}^{\prime} \bar{\gamma}_{2}\right)(t)=i e^{i \mu t} \bar{\gamma}_{1}(t)^{n_{1}+1} \bar{\gamma}_{2}(t)^{n_{2}+1} .
$$

This Legendrian immersion $\phi$ is minimal if and only if $\psi_{1}$ and $\psi_{2}$ are minimal and $\left(\gamma_{1}, \gamma_{2}\right)$ is a solution of (24) with $\mu=0$.

If we consider the particular cases $n_{2}=0$ and $n_{1}=0$ in the minimal case of Corollary 6.5, we recover (projecting via the Hopf fibration $\Pi$ ) the minimal Lagrangian submanifolds of $\mathbb{C} \mathbb{T}^{n}$ described in [Castro et al. 2002, Propositions 3 and 5], although we used there a unit speed parametrization for $\gamma$.

From these two last results we can get similar examples to the ones given in Section 4 in the projective case. Concretely, it is easy to check that for any $\rho>0$ the Legendre curve

$$
\gamma_{\rho}(t)=\left(s_{\rho} \exp \left(i s_{\rho}^{n_{1}-1} c_{\rho}^{n_{2}+1} t\right), c_{\rho} \exp \left(i s_{\rho}^{n_{1}+1} c_{\rho}^{n_{2}-1} t\right)\right),
$$

satisfies (24) for $\mu=s_{\rho}^{n_{1}-1} c_{\rho}^{n_{2}-1}\left(\left(n_{1}+1\right) c_{\rho}^{2}+\left(n_{2}+1\right) s_{\rho}^{2}\right)$, where $c_{\rho}=\cosh \rho$, $s_{\rho}=\sinh \rho$.

Hence an analogous reasoning to that in Corollary 4.1 yields following explicit family of examples.

Corollary 6.6. Let $\psi_{1}: N_{1} \rightarrow \mathbb{S}^{2 n_{1}+1} \subset \mathbb{C}^{n_{1}+1}$ and $\psi_{2}: N_{2} \rightarrow \mathbb{M}_{1}^{2 n_{2}+1} \subset \mathbb{C}^{n_{2}+1}$ be $C$-minimal Legendrian immersions of $n_{i}$-dimensional Riemannian manifolds $N_{i}$, $i=1,2$, and let $n=n_{1}+n_{2}+1$. Given $\rho>0$, set $c_{\rho}=\cosh \rho$ and $s_{\rho}=\sinh \rho$. Then the map $\Phi_{\rho}: \mathbb{S}^{1} \times N_{1} \times N_{2} \rightarrow \mathbb{C}^{n}$ given by

$$
\Phi_{\rho}\left(e^{i t}, p, q\right)=\left[\left(s_{\rho} \exp \left(i t c_{\rho}^{2}\right) \psi_{1}(p), c_{\rho} \exp \left(i t s_{\rho}^{2}\right) \psi_{2}(q)\right)\right]
$$

is a H-minimal Lagrangian immersion.

A particular case of Corollary 6.6 gives a one-parameter family of H-minimal Lagrangian embeddings.

Corollary 6.7. Let $\rho>0$ and $n=n_{1}+n_{2}+1$. The immersion $\Phi_{\rho}$ of Corollary 6.6, where $\psi_{1}$ (resp. $\left.\psi_{2}\right)$ is the totally geodesic Legendrian embedding of $\mathbb{S}^{n_{1}}$ into $\mathbb{S}^{2 n_{1}+1}$ (resp. of $\mathbb{R} \mathbb{H}^{n_{2}}$ into $\mathbb{\boxplus}_{1}^{2 n_{2}+1}$ ), provides a $H$-minimal Lagrangian embedding

$$
\overline{\left(e^{i t}, x, y\right)} \mapsto\left[\left(s_{\rho} \exp \left(i t c_{\rho}^{2}\right) x, c_{\rho} \exp \left(i t s_{\rho}^{2}\right) y\right)\right]
$$

of the quotient of $\mathbb{S}^{1} \times \mathbb{S}^{n_{1}} \times \mathbb{R} \mathbb{M}^{n_{2}}$ by the action of the group $\mathbb{Z}_{2}$ into $\mathbb{C} \mathbb{H}^{n}$, the action of $\mathbb{Z}_{2}$ being generated by the involution $\left(e^{i s}, x, y\right) \mapsto\left(-e^{i s},-x, y\right)$. 
We finally turn our attention to (24) with $\mu=0$. We observe that this is exactly equation (3) in [Castro and Urbano 2004, Lemma 2] (with $p=n_{1}$ and $q=n_{2}$ ). If we choose the initial conditions $\gamma(0)=(\sinh \varrho, \cosh \varrho), \varrho>0$, we can make use of the study made in that paper.

Lemma 6.8. Let $\gamma_{\varrho}=\left(\gamma_{1}, \gamma_{2}\right): I \subset \mathbb{R} \rightarrow \mathbb{H}_{1}^{3}$ be the unique curve solution of

$$
\gamma_{j}^{\prime} \bar{\gamma}_{j}=i \bar{\gamma}_{1}^{n_{1}+1} \bar{\gamma}_{2}^{n_{2}+1}, j=1,2,
$$

satisfying the real initial conditions $\gamma_{\varrho}(0)=(\sinh \varrho, \cosh \varrho), \varrho>0$.

(1) $\operatorname{Re}\left(\gamma_{1}^{n_{1}+1} \gamma_{2}^{n_{2}+1}\right)=\sinh ^{n_{1}+1} \varrho \cosh ^{n_{2}+1} \varrho$.

(2) For $j=1,2$ and any $t \in I$, we have $\bar{\gamma}_{j}(t)=\gamma_{j}(-t)$.

(3) The curves $\gamma_{1}$ and $\gamma_{2}$ are embedded and can be parametrized by $\gamma_{j}(t)=$ $\rho_{j}(t) e^{i \theta_{j}(t)}$, where we have set $\left(\right.$ with $\left.c_{\varrho}=\cosh \varrho, s_{\varrho}=\sinh \varrho\right)$

$$
\begin{aligned}
& \rho_{1}(t)=\sqrt{t^{2}+s_{\varrho}^{2}}, \\
& \theta_{1}(t)=\int_{0}^{t} \frac{s_{\varrho}^{n_{1}+1} c_{\varrho}^{n_{2}+1} x d x}{\left(x^{2}+s_{\varrho}^{2}\right) \sqrt{\left(x^{2}+s_{\varrho}^{2}\right)^{n_{1}+1}\left(x^{2}+c_{\varrho}^{2}\right)^{n_{2}+1}-s_{\varrho}^{2\left(n_{1}+1\right)} c_{\varrho}^{2\left(n_{2}+1\right)}}}, \\
& \rho_{2}(t)=\sqrt{t^{2}+c_{\varrho}^{2}}, \\
& \theta_{2}(t)=\int_{0}^{t} \frac{s_{\varrho}^{n_{1}+1} c_{\varrho}^{n_{2}+1} x d x}{\left(x^{2}+c_{\varrho}^{2}\right) \sqrt{\left(x^{2}+s_{\varrho}^{2}\right)^{n_{1}+1}\left(x^{2}+c_{\varrho}^{2}\right)^{n_{2}+1}-s_{\varrho}^{2\left(n_{1}+1\right)} c_{\varrho}^{2\left(n_{2}+1\right)}}} .
\end{aligned}
$$

In this way, the immersions $\phi_{\varrho}$ constructed with the curves $\gamma_{\varrho}$ of Lemma 6.8 induce a one-parameter family of $\mathrm{H}$-minimal Lagrangian immersions

$$
\Phi_{\varrho}: \mathbb{R} \times N_{1} \times N_{2} \rightarrow \mathbb{C}^{n}, n=n_{1}+n_{2}+1, \varrho>0 .
$$

In particular, $\Phi_{\varrho}$ is minimal if and only if $\psi_{1}$ and $\psi_{2}$ are minimal. We conclude with the following particular case, which leads to a one-parameter family of minimal Lagrangian embeddings.

Corollary 6.9. Let $\varrho>0$ and $\operatorname{set} c_{\varrho}=\cosh \varrho, s_{\varrho}=\sinh \varrho$. Then

$$
\begin{gathered}
\mathbb{R} \times \mathbb{S}^{n_{1}} \times \mathbb{R} \mathbb{M}^{n_{2}} \rightarrow \mathbb{C} \mathbb{H}^{n}, n=n_{1}+n_{2}+1, \\
(s, x, y) \mapsto\left[\left(\sqrt{s^{2}+s_{\varrho}^{2}} \exp \left(i \theta_{1}(s)\right) x, \sqrt{s^{2}+c_{\varrho}^{2}} \exp \left(i \theta_{2}(s)\right) y\right)\right],
\end{gathered}
$$

where the $\theta_{i}(s)$ are given in Lemma 6.8(3), is a minimal Lagrangian embedding.

Acknowledgements. The authors thank the referee for helpful comments and suggestions. 


\section{References}

[Anciaux 2003] H. Anciaux, "Construction of many Hamiltonian stationary Lagrangian surfaces in Euclidean four-space", Calc. Var. Partial Diff. Equations 17:2 (2003), 105-120. MR 2004i:53118 Zbl 1042.53004

[Anciaux et al. 2006] H. Anciaux, I. Castro, and P. Romon, "Lagrangian submanifolds foliated by $(n-1)$-spheres in $\mathbb{R}^{2 n}$, Acta Math. Sinica 22:4 (2006), 1197-1214.

[Castro and Urbano 1998] I. Castro and F. Urbano, "Examples of unstable Hamiltonian-minimal Lagrangian tori in $\mathbb{C}^{2}$ ", Compositio Math. 111:1 (1998), 1-14. MR 98m:53075 Zbl 0896.53039

[Castro and Urbano 2004] I. Castro and F. Urbano, "On a new construction of special Lagrangian immersions in complex Euclidean space”, Q. J. Math. 55:3 (2004), 253-265. MR 2005g:53087 Zbl 02138381

[Castro et al. 2001] I. Castro, C. R. Montealegre, and F. Urbano, "Closed conformal vector fields and Lagrangian submanifolds in complex space forms", Pacific J. Math. 199:2 (2001), 269-302. MR 2002g:53137 Zbl 1057.53063

[Castro et al. 2002] I. Castro, C. R. Montealegre, and F. Urbano, "Minimal Lagrangian submanifolds in the complex hyperbolic space", Illinois J. Math. 46:3 (2002), 695-721. MR 2004e:53089 Zbl 1032.53052

[Eells and Ratto 1993] J. Eells and A. Ratto, Harmonic maps and minimal immersions with symmetries, Annals of Mathematics Studies 130, Princeton University Press, Princeton, NJ, 1993. MR 94k:58033 Zbl 0783.58003

[Haskins 2004a] M. Haskins, "The geometric complexity of special Lagrangian $T^{2}$-cones", Invent. Math. 157:1 (2004), 11-70. MR 2005m:53085 Zbl 1064.53032

[Haskins 2004b] M. Haskins, "Special Lagrangian cones”, Amer. J. Math. 126:4 (2004), 845-871. MR 2005e:53074 Zbl 1074.53067

[Hélein and Romon 2000] F. Hélein and P. Romon, "Weierstrass representation of Lagrangian surfaces in four-dimensional space using spinors and quaternions", Comment. Math. Helv. 75:4 (2000), 668-680. MR 2001m:53102 Zbl 0973.53065

[Hélein and Romon 2002a] F. Hélein and P. Romon, "Hamiltonian stationary Lagrangian surfaces in $\mathbb{C}^{2}$,, Comm. Anal. Geom. 10:1 (2002), 79-126. MR 2003e:53108 Zbl 1007.53060

[Hélein and Romon 2002b] F. Hélein and P. Romon, "Hamiltonian stationary Lagrangian surfaces in Hermitian symmetric spaces", pp. 161-178 in Differential geometry and integrable systems (Tokyo, 2000), edited by M. Guest et al., Contemp. Math. 308, Amer. Math. Soc., Providence, RI, 2002. MR 2004j:53103 Zbl 1036.53058

[Hélein and Romon 2003] F. Hélein and P. Romon, "Hamiltonian stationary tori in complex projective plane", preprint, 2003. math.DG/0310095

[McDuff and Salamon 1998] D. McDuff and D. Salamon, Introduction to symplectic topology, Oxford University Press, New York, 1998. MR 2000g:53098 Zbl 1066.53137

[Mironov 2004] A. E. Mironov, "New examples of Hamilton-minimal and minimal Lagrangian submanifolds in $\mathbb{C}^{n}$ and $\mathbb{C P}^{n}$ ", Mat. Sb. 195:1 (2004), 89-102. In Russian; translated in Sb. Math. 195:1 (2004), 85-96. MR 2005e:53127 Zbl 1078.53079

[Montealegre and Vrancken 2006] C. R. Montealegre and L. Vrancken, "Pinching properties of Lagrangian submanifolds of complex projective space", preprint, 2006.

[Naitoh 1981] H. Naitoh, "Isotropic submanifolds with parallel second fundamental form in $P^{m}(c)$ ", Osaka J. Math. 18:2 (1981), 427-464. MR 83b:53051 Zbl 0471.53036 
[Oh 1990] Y.-G. Oh, "Second variation and stabilities of minimal Lagrangian submanifolds in Kähler manifolds", Invent. Math. 101:2 (1990), 501-519. MR 91f:58022 Zbl 0721.53060

[Schoen and Wolfson 1999] R. Schoen and J. Wolfson, "Minimizing volume among Lagrangian submanifolds", pp. 181-199 in Differential equations: La Pietra 1996 (Florence, 1996), edited by M. Giaquinta et al., Proc. Sympos. Pure Math. 65, Amer. Math. Soc., Providence, RI, 1999. MR 99k:53130 Zbl 1031.53112

Received December 1, 2004. Revised June 29, 2005.

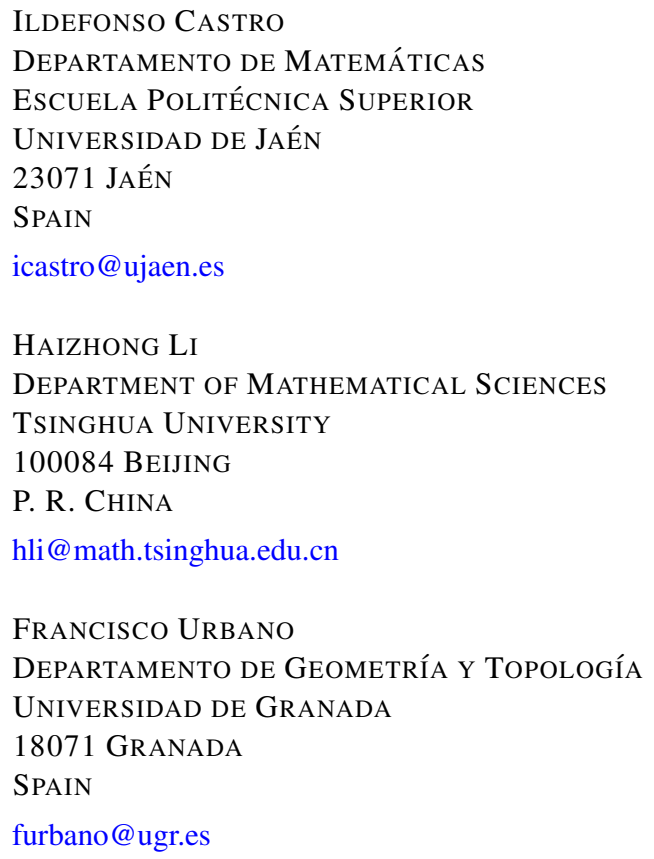

